\title{
Diluted planar ferromagnets: nonlinear excitations on a non-simply connected manifold
}

\author{
Fagner M. Paula, Afranio R. Pereira $⿴$ and Lucas A. S. Móđ \\ Departamento de Física, Universidade Federal de Viçosa, Viçosa, 36570-000, Minas Gerais, Brazil.
}

\begin{abstract}
We study the behavior of magnetic vortices on a two-dimensional support manifold being not simply connected. It is done by considering the continuum approach of the XY-model on a plane with two disks removed from it. We argue that an effective attractive interaction between the two disks may exist due to the presence of a vortex. The results can be applied to diluted planar ferromagnets with easy-plane anisotropy, where the disks can be seen as nonmagnetic impurities. Simulations are also used to test the predictions of the continuum limit.
\end{abstract}

PACS numbers: 75.10. Hk; 75.10.-b; 05.45.Yv;

Keywords: Impurities, Vortices, XY-model.

Physics in two spatial dimensions has generated a lot of surprises. In condensed matter systems these surprises are specially interesting due to the possibility of technological applications. The role of nonlinear excitations in the study of low-dimensional, artificially structured materials is a very exciting topic due to their observable effects on the physical properties of a realizable condensed matter system. Nonconventional supports (such as curved surfaces) for condensed matter materials may induce a much richer physics because the interplay between geometry and topology [1, 2, 3, 4]. Coulomb systems living on a sphere [5, 6], magnetic systems living on a cylinder or a sphere [1, 2, 3, 4] etc have already been considered recently. Even flat surfaces (the plane $R^{2}$ ) with pieces cut out from them open up new, interesting avenues of investigation. For example, if one or more sectors are excised from a single layer of graphite and the remainder is joined seamlessly, a cone results [7, 8]. By considering the symmetry of a graphite sheet and the Euler's theorem, it can be shown that only five types of cone can be made from a continuous sheet of graphite corresponding to the following values of cone angles $\gamma=19.2^{\circ}, 38.9^{\circ}, 60.0^{\circ}, 84.6^{\circ}, 112.9^{\circ}$. These are all synthesized forming carbon nanocones [8]. The conical geometry is also interesting because of its connection with the problem of Einstein gravity in low dimensions [9]. Another interesting possibility is the plane $R^{2}$ with a disk of radius $a$ cut out from it. Such a space is said to be not simply connected since it has the property that some closed curves drawn in it can not be continuously shrunk to a point. In two-dimensional magnetic materials described by the continuum limit, such a disk cut out from the plane has a simple physical interpretation. It can be seen as a nonmagnetic impurity present into the system [10, 11, 12, 13]. Then, from now, it is convenient to use the language of magnetism.

\footnotetext{
* Corresponding author : Afranio Rodrigues Pereira.

Tel.: 55-31-3899-2988; fax: 55-31-3899-2483.; Electronic address: apereira@ufv.br

${ }^{\dagger}$ Electronic address: lucasmol@ufv.br
}

A large variety of two-dimensional magnetic materials are well described by the anisotropic Heisenberg models. Particularly, many of these materials are modelled as a continuum of classical spins with easy-plane symmetry. In these systems, vortices are important excitations, responsible for interesting static and dynamic properties [14, 15, 16, 17]. In this letter, we explore the effect of the support manifold (the plane $R^{2}$ ) not simply connected and focus on the vortex behavior. Specifically, we consider the plane $R^{2}$ with two disks of radius $a$ cut out from it. In the case of magnetism, the size $a$ is the lattice constant. We note, however, that in experimental situations involving small scales (e.g., a small hole with size of the order of lattice spacing $a$ ) the applications of the continuum approximation (i.e., a long wavelength theory) should be viewed with caution. Then, we also compare some results of this theory with numerical simulations on a discrete lattice. Our main motivation is to know how magnetic vortices could be pinned in the system. Recent works 10, 11, 12, 13] have shown that the topological excitations center is attracted and pinned by a nonmagnetic impurity. However, a real magnetic material contains many vacancies and then, the possibility of pinned vortices not centered on vacancies but among them should be investigated. Here, first we consider two spin vacancies and a single vortex to calculate the equilibrium position for the vortex center. The configurations of energy minima are then obtained. It is shown that the effective potential experienced by a vortex due to the presence of the two lattice defects attracts the vortex center to either one of the vacancies center or the center of the line joining the two vacancies. Second, we argue that the presence of the magnetic vortex background may induce an effective interaction between the holes that is attractive. This interaction results in a geometrical frustration in the region between vacancies.

The easy-plane ferromagnets are described by the Hamiltonian

$$
H=-J \sum_{i, j}\left[S_{i}^{x} S_{j}^{x}+S_{i}^{y} S_{j}^{y}+\lambda S_{i}^{z} S_{j}^{z}\right],
$$

where $J>0$ is the exchange constant, $0 \leq \lambda<1$ is 
the easy-plane anisotropy and $\vec{S}_{i}=\left\{S_{i}^{x}, S_{i}^{y}, S_{i}^{z}\right\}$ is the classical spin vector at site $i$. We focus on the case $\lambda=0$ (XY-model). The spin field can be parametrized by two scalar fields $\phi$ and $m=\cos \theta$ (the azimuthal and polar angles) as follows $\vec{S}=\left\{\sqrt{1-m^{2}} \cos \phi, \sqrt{1-m^{2}} \sin \phi, m\right\}$. The effective interaction among a single vortex and the two spinless sites is discussed by using a simple continuum XY-Hamiltonian on a support manifold being not simply connected, written as

$$
\begin{gathered}
H_{I}=\frac{J}{2} \int\left[\frac{m^{2}(\vec{\nabla} \phi)^{2}}{1-m^{2}}+\left(1-m^{2}\right)(\vec{\nabla} \phi)^{2}+\frac{4}{a^{2}} m^{2}\right] \times \\
U_{1}(\vec{r}) U_{2}(\vec{r}) d^{2} r
\end{gathered}
$$

where the term in brackets is the Hamiltonian density $\mathrm{H}$ for the continuum version of pure Hamiltonian (1), the function $U_{1}(\vec{r})$ defined as $U_{1}(\vec{r})=1$ if $\left|\vec{r}-\vec{r}_{1}\right| \geq a$ and $U_{1}(\vec{r})=0$ if $\left|\vec{r}-\vec{r}_{1}\right|<a$, represents the spin vacancy (the first disk) centered at position $\vec{r}_{1}$ and the function $U_{2}(\vec{r})$ defined as $U_{2}(\vec{r})=1$ if $\left|\vec{r}-\vec{r}_{2}\right| \geq a$ and $U_{2}(\vec{r})=0$ if $\left|\vec{r}-\vec{r}_{2}\right|<a$, represents another spin vacancy (the second disk) centered at position $\vec{r}_{2}$. The distance between vacancies is $p=\left|\vec{r}_{2}-\vec{r}_{1}\right|$. We now make a very simple mathematical observation that follows from the associative property of the terms present in expression (2) and that justify the equivalency between a hole on the plane and a vacancy in the Hamiltonian. Note that $\int \mathrm{H}\left[U_{1}(\vec{r}) U_{2}(\vec{r}) d^{2} r\right]=\int\left[\mathrm{H} U_{1}(\vec{r}) U_{2}(\vec{r})\right] d^{2} r$ and then, the problem of a magnetically coated plane not simply connected (left side of the identity) is equivalent to the problem of a magnetic plane simply connected but containing spin vacancies in the Hamiltonian (right side). It only depends on the point of view: the disks are removed either from the plane or from the Hamiltonian density. With this in mind, we remember that simulations show that the vortex structure is not deformed appreciably due the presence of a hole [13]. It will also be assumed in our calculations.

The XY-model supports only planar vortices [19], which the configuration is $m_{v}=0, \phi_{v}=\arctan [(y-$ $\left.\left.y_{v}\right) /\left(x-x_{v}\right)\right]$ for the vortex center localized at $\left(x_{v}, y_{v}\right)$. The energy of this vortex configuration, in the absence of nonmagnetic impurities, is $E_{v}=\pi J \ln (L / 0.24 a)$ [19], where $L$ is the system size. Then, considering the vortex center localized at origin (for simplicity), the effective interaction between the vortex and the pair of impurities is given by $V_{\text {eff }}\left(\vec{r}_{1}, \vec{r}_{2}\right)=E\left(\vec{r}_{1}, \vec{r}_{2}\right)-E_{v}$, where $E\left(\vec{r}_{1}, \vec{r}_{2}\right)$ is the vortex energy in the presence of the two impurities placed at $\vec{r}_{1}$ and $\vec{r}_{2}$ respectively. Using Hamiltonian (2), the planar vortex energy in the presence of two holes (nonmagnetic impurities) is simply obtained leading to

$$
\begin{gathered}
E\left(\vec{r}_{1}, \vec{r}_{2}\right)=E_{v}+\frac{\pi J}{2} \ln \left[1-\frac{a^{2}}{r_{1}^{2}+d^{2}}-\frac{a^{2}}{r_{2}^{2}+d^{2}}+\right. \\
\left.\frac{a^{4}}{\left(r_{1}^{2}+d^{2}\right)\left(r_{2}^{2}+d^{2}\right)}\right]
\end{gathered}
$$

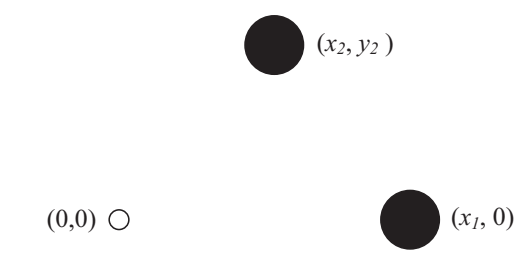

FIG. 1: A vortex (white circle) located at origin $(0,0)$ near two holes (black circles) located at $\vec{r}_{1}=\left(x_{1}, 0\right)$ and $\vec{r}_{2}=$ $\left(x_{2}, y_{2}\right)$. The distance between the holes is $p=\left|\vec{r}_{2}-\vec{r}_{1}\right|$.

where $d$ is a suitable constant of the order of the lattice spacing $a$ introduced in Eq.(3) in order to avoid spurious divergences of the vortex energy (in the continuum approach) when the vortex center coincides with an impurity center. Thus, to find this constant, we note that, in the case in which the centers of the three defects (two defects in the lattice and one in the spin field) are located at the same point (for example, at origin), then, the three "bodies" problem reduces to a simpler problem of a vortex on a single hole. In this case, $V_{\text {eff }}(\overrightarrow{0}, \overrightarrow{0})$ obtained from Eq.(3), should have the value $-4.48 J$ (see Ref. 13]) and hence, we get $d=1.1472 a$. Although the limit that the separation vanishes is reasonable in the continuum approach, in a real discrete lattice two vacancies can not occupy the same point. Therefore, these approximations can give us important insights about the system with topological excitations and nonmagnetic impurities.

The object now is to calculate the configurations of minimum energy involving these three defects. It means that we need to minimize the quantity

$F_{d}\left(r_{1}, r_{2}\right)=-\frac{1}{r_{1}^{2}+d^{2}}-\frac{1}{r_{2}^{2}+d^{2}}+\frac{a^{2}}{\left(r_{1}^{2}+d^{2}\right)\left(r_{2}^{2}+d^{2}\right)} .(4)$

To do this, we consider the geometry shown in Fig.(1). In this figure, the vortex center is located at the origin and the coordinates of the nonmagnetic impurities are $\left(x_{1}, 0\right)$ and $\left(x_{2}, y_{2}\right)$. Here we have chosen $y_{1}=0$ (i.e., the hole 1 located along the x-axis) for effect of simplicity. Then, Eq.(4) is rewritten as

$$
F_{d}\left(x_{1}, x_{2}, y_{2}\right)=\frac{a^{2}-2 d^{2}-x_{1}^{2}-x_{2}^{2}-y_{2}^{2}}{\left(x_{1}^{2}+d^{2}\right)\left(x_{2}^{2}+y_{2}^{2}+d^{2}\right)}
$$

Using the Lagrangian multipliers method, one has $\vec{\nabla} F_{d}\left(x_{1}, x_{2}, y_{2}\right)=-\kappa \vec{\nabla} \varphi\left(x_{1}, x_{2}, y_{2}\right)$, with $\varphi\left(x_{1}, x_{2}, y_{2}\right)=$ $p^{2}-\left(x_{2}-x_{1}\right)^{2}-y_{2}^{2}=0$, where $\varphi$ is a constraint that keeps invariable the distance $p$ between the two vacancies and $\kappa$ is a parameter. Thus, we get the following system 
of equations to be solved for $x_{1}, x_{2}$ and $y_{2}$

$$
\left\{\begin{array}{c}
x_{1} \frac{\left(a^{2}-d^{2}-x_{2}^{2}-y_{2}^{2}\right)}{\left(x_{1}^{2}+d^{2}\right)^{2}\left(x_{2}^{2}+y_{2}^{2}+d^{2}\right)}-\kappa\left(x_{2}-x_{1}\right)=0 \\
x_{2} \frac{\left(a^{2}-d^{2}-x_{1}^{2}\right)}{\left(x_{1}^{2}+d^{2}\right)\left(x_{2}^{2}+y_{2}^{2}+d^{2}\right)^{2}}-\kappa\left(x_{2}-x_{1}\right)=0 \\
y_{2}\left[\frac{a^{2}-d^{2}-x_{1}^{2}}{\left(x_{1}^{2}+d^{2}\right)\left(x_{2}^{2}+y_{2}^{2}+d^{2}\right)^{2}}+\kappa\right]=0 \\
p^{2}-\left(x_{2}-x_{1}\right)^{2}-y_{2}^{2}=0
\end{array}\right.
$$

We found two solutions for the above equations that satisfy our requirement of extremum for $F_{d}$ :

$$
\left(x_{1}, x_{2}, y_{2}\right)=\left( \pm \frac{p}{2}, \mp \frac{p}{2}, 0\right)
$$

or

$$
\left(x_{1}, x_{2}, y_{2}\right)=(0,0, \pm p)
$$

The identification of these solutions as energy minima will follow from a consideration of the original equations. Substituting Eqs. (7) and (8) into Eq. (3), one obtains the two energies $E_{1 v}$ and $E_{2 v}$ as a function of $p$ for these configurations, respectively

$$
E_{1 v}=E_{v}+\frac{\pi J}{2} \ln \left(1-\frac{a^{2}}{(p / 2)^{2}+d^{2}}\right)^{2}
$$

and

$$
E_{2 v}=E_{v}+\frac{\pi J}{2} \ln \left[\left(1-\frac{a^{2}}{p^{2}+d^{2}}\right)\left(1-\frac{a^{2}}{d^{2}}\right)\right] .
$$

Hence, in general, any configuration of these three defects in which the vortex center is found at the central point of the line joining the two impurity centers, leads to the situation with energy $E_{1 v}$. By the other side, configurations of this system in which the vortex center is found at the center of one of the two impurities, produce the energy $E_{2 v}$.

Now, it should be interesting to check the conditions of stability of these two possibilities. It can be done by comparing the energies $E_{1 v}$ and $E_{2 v}$ as a function of the impurities separation $p$. Figure (2) shows the functions $\xi_{i}(p)$, defined as $\xi_{i}(p)=E_{i v}-E_{v},(i=1,2)$. Note that $\xi_{1 v}>\xi_{2 v}$ for any $p>1.087 a$ and $\xi_{1 v}<\xi_{2 v}$ for $0<p<1.087 a$. It indicates that configuration 2 (with the vortex center coinciding with an impurity center) is energetically favorable for an appreciable range of impurities separation $p$. It is interesting to note that, in the case of the two nearest neighbor vacancies (separated by the distance $a$ ), the vortex center should occupy the center of the intersection of holes (or the center of the large plaquette containing the two neighbor spinless sites in a discrete lattice). Figure 2 also shows that the potential $\xi_{2}$ is practically constant for $p>2 a$, and hence

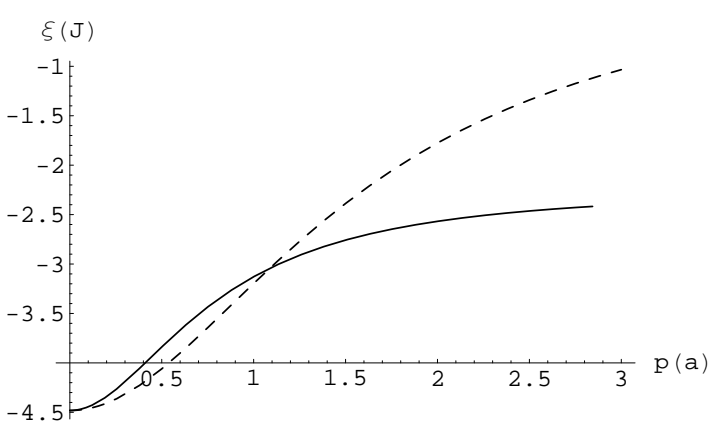

FIG. 2: Potentials $\xi_{1}$ (dashed curve) and $\xi_{2}$ (solid curve) versus $p$. For $p>1.087 a, \xi_{2}<\xi_{1}$.

the force between the vortex-on-vacancy and the other vacancy decays rapidly with the distance of separation. Note also that, in this case, the energy necessary to remove a vortex from a vacancy is $2.24 J$, which is smaller than $4.48 \mathrm{~J}$, indicating that the presence of other impurities lower the biding energy of the vortex-on-vacancy state. Extrapolating the present results for quenched diluted layered magnetic materials with very low impurity concentrations (in this case it is expect that the vortex density is almost the same as the vacancy density), it is conceivable that a magnetic vortex lattice (not necessarily periodic, since the vortices distribution would be alike the vacancies distribution) could be present into the system. Besides, this vortex lattice could not be so rigid, because, as we have seen, the impurity concentration must decrease the vortex-on-vacancy binding energy, giving some mobility to vortices. This picture would have important consequences to the spin dynamics, which may be observed in experiments. For example, the neutron scattering function $S_{\gamma \gamma}(\vec{q}, \omega)$ may still exhibit a central peak (the cause of this peak is believed to be the vortex translational motion [15, 16, 17]), but its shape, width and height should modify considerably.

Next, we investigate the nature of the interaction between two static vacancies through the vortex background. In Ref. [13], it was shown that the energy of a vortex at origin in the presence of only one impurity at distance $\left|\vec{r}_{i}\right|$ away is given by $E_{v}+(\pi J / 2) \ln \left[1-a^{2} /\left(r_{i}^{2}+\right.\right.$ $\left.b^{2}\right)$ ], where $b=1.03 a$. Hence, the energy cost of removing one spin (placed at $\vec{r}_{i}$ ) from an infinite plane containing a vortex at origin is $\epsilon_{1}\left(\vec{r}_{i}\right)=-(\pi J / 2) \ln \left[1-a^{2} /\left(r_{i}^{2}+b^{2}\right)\right]$. Of course, due to the cylindrical symmetry, this energy does not depend on the direction of $\vec{r}_{i}$. From Eq. (3), it is easy to see that the energy cost of removing two spins (located at sites $\vec{r}_{1}$ and $\vec{r}_{2}$ ) from the vortex background is

$$
\begin{array}{r}
\epsilon_{2}\left(\vec{r}_{1}, \vec{r}_{2}\right)=-\frac{\pi J}{2} \ln \left[1-\frac{a^{2}}{r_{1}^{2}+d^{2}}-\frac{a^{2}}{r_{2}^{2}+d^{2}}+\right. \\
\left.\frac{a^{4}}{\left(r_{1}^{2}+d^{2}\right)\left(r_{2}^{2}+d^{2}\right)}\right] .
\end{array}
$$


Thus, there is an effective interaction potential between two static holes through the vortex background given by $\Delta=\epsilon_{2}\left(\vec{r}_{1}, \vec{r}_{2}\right)-\epsilon_{1}\left(\vec{r}_{1}\right)-\epsilon_{1}\left(\vec{r}_{2}\right)$. This potential is, then, expressed as

$$
\Delta=\frac{\pi J}{2} \ln \left[\frac{1+a^{2} F_{b}\left(r_{1}, r_{2}\right)}{1+a^{2} F_{d}\left(r_{1}, r_{2}\right)}\right]
$$

where $F_{b}\left(r_{1}, r_{2}\right)$ is also given by expression (4) but with $d$ substituted by $b$. This effective interaction depends on the distance of separation as well as the orientation of vacancies in the vortex background. Since $b<d$, this energy is negative, which means that the effective interaction between vacancies is attractive. The problem can be summarized as follows: consider an initial configuration of these three defects as shown, for example, in Fig. (1). Supposing that $p>1.087 a$, the spin distribution (or the magnetic energy) will be then readjusted to minimize the system energy, making the vortex center to move to the nearest vacancy center. However, the spin distribution did not reach its minimum in energy yet. The vortex-on-vacancy is still attracted by the other vacancy, but now, neither the vortex-on-vacancy state nor the other vacancy can dislocate on the plane. In fact, in a rigid plane (lattice), the holes are fixed to their original positions and can not move on the plane. As a consequence, the system cannot reach its minimum in energy anymore; there is a geometrical frustration, which stems from a constraint (an invariable distance $p$ between the two disconnected holes). Of course, the static-vacancy problem is an important limiting model for understanding the interaction of mobile holes through the magnetic background. If the vacancies were mobile, an attractive interaction could result in holes pairing around the magnetic plane. Probably, in an elastic support, a variation in the geometry of the plane, compensating for the constraint, may lead to a lowering of the energy. It is expected that if the rigidity condition were relaxed, an elastic Hamiltonian density introduced in the problem should stabilize the plane against arbitrary deformations.

To test some results obtained by using the continuum limit, we have also performed spin dynamical simulations on a $L=20 a$ square lattice to study the behavior of a single vortex with its center initially located at the center of the system $(0,0)$, in the presence of two spin vacancies located at $(a / 2,-3 a / 2)$ and $(-a / 2,3 a / 2)$ respectively (see Fig. (3)). This configuration starts with the vortex localized exactly at the central point of the line joining the two vacancies. Note that the analytical results indicate that it is an unstable situation, since $p \approx 3.16 a$, which is bigger than 1.087a. Then, it is expected that, in the simulations, the vortex center must move in direction to one of the spinless sites. To see this we have imposed diagonally antiperiodic boundary conditions [20] $\vec{S}_{L+1, y}=-\vec{S}_{1, L-y+1}, \vec{S}_{0, y}=-\vec{S}_{L, L-y+1}$ and $\vec{S}_{x, L+1}=\vec{S}_{L-x+1,1}, \quad \vec{S}_{x, 0}=-\vec{S}_{L-x+1, L}$, for all $1 \leq x, y \leq L$, in order to keep the vortex structure stable. The discrete motion equation for each spin is given by [21]

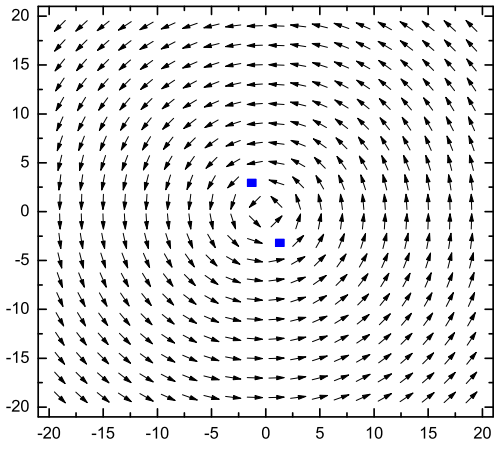

FIG. 3: Initial configuration with a vortex located at origin and two vacancies at $(-a / 2,3 a / 2)$ and $(a / 2,-3 a / 2)$ respectively. The squares represent the spinless sites and the distance between them is $p=3.16 a$. The length scale is expressed in units of $a / 2$. Many other configurations were also studied confirming the analytical results.

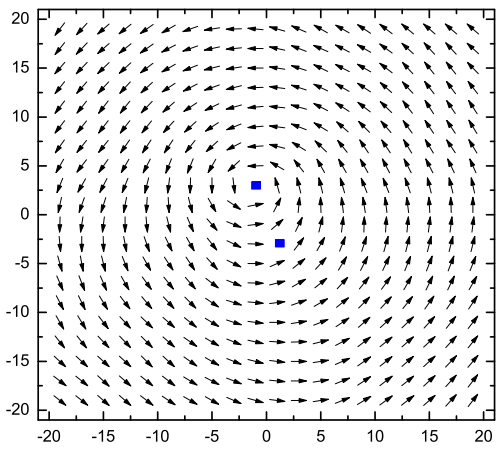

FIG. 4: Configuration after $7 \times 10^{4}$ time steps. The vortex travelled from the central position between vacancies to one of the vacancies center. It is in agreement with analytical results.

$d \vec{S}_{i} / d t=\vec{S}_{i} \times \vec{h}$, where $\vec{h}=-J \sum_{j}\left(S_{j}^{x} \hat{e}_{x}+S_{j}^{y} \hat{e}_{y}\right)$ and $\hat{e}_{x}$ and $\hat{e}_{y}$ are unit vectors in the $x$ and $y$ directions, respectively. The motion equations were integrated numerically forward in time using a fourth-order Runge-Kutta scheme with a time step of $4 \times 10^{-4} J^{-1}$. We notice that after $10^{4}$ time steps the position of the vortex center always reaches one of the impurity centers (Fig. (4)). On the other hand, if the holes are separated by one lattice spacing, the continuum theory predicts that the vortex center should stay on the central point joining the holes. Our simulations also agree with this result as it can be seen in figures 5 and 6 . Figure (5) shows an initial configuration with a vortex at origin and two neighbor vacancies at $(3 a / 2, a / 2)$ and $(5 a / 2, a / 2)$ respectively. After $10^{4}$ time 


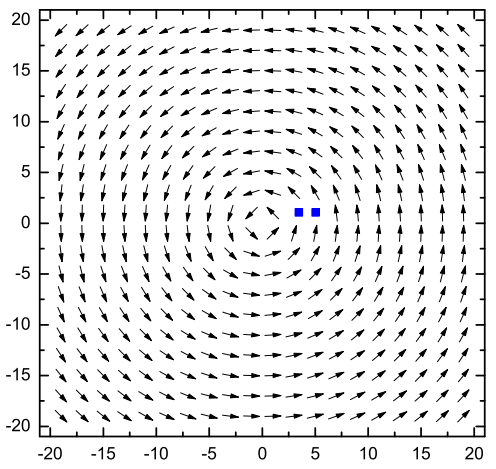

FIG. 5: Initial configuration with a vortex located at origin and two vacancies at $(3 a / 2, a / 2)$ and $(5 a / 2, a / 2)$ respectively. The distance between these two vacancies is $1 a$.

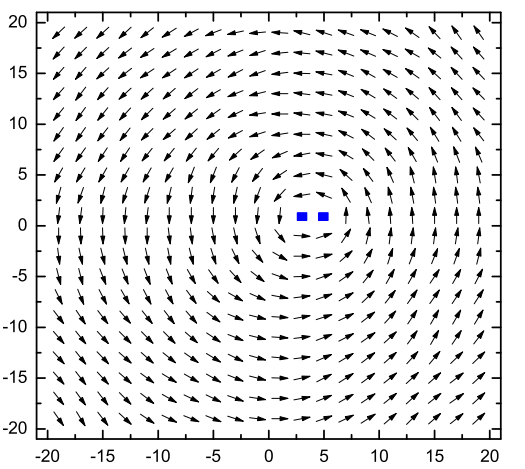

FIG. 6: Final state of Fig.(5). Now, the vortex center stays in the central point of the line joining the two vacancies in agreement with analytical results. steps, the vortex center stays between the vacancies (Fig. $(6))$. However, there is the possibility that this last configuration may not be completely static, but the vortex center seems to oscillate around the central point joining the vacancies, affecting the spin dynamics. The analysis of this possibility is out of the scope of this work and will be investigated in a future paper.

In summary, we have studied the problem of the magnetic vortex behavior in a non-simply connected manifold. The results can be applied to magnetic materials, including antiferromagnetic systems. The flat plane considered here was constructed by cutting two holes, which can be interpreted as nonmagnetic impurities. The cut edges (which are the boundary surfaces for the infinite two-dimensional plane) exert a profound influence on the vortex center. There are two ways of pinning a vortex, in which the stability depends on the distance $p$ between the holes. Besides, we have shown that the vortex background induces an effective interaction potential between two vacancies that is attractive. It generates geometrical frustration. If the holes and the resulting hole-vortex hybrids have metallic mobilities, then such an effective interaction could be a pairing mechanism for superconductivity in doped layered antiferromagnets. The overall qualitative agreement between the analytical results using the continuum approach on a plane with two holes and the simulations on a discrete lattice with two missing spins is striking. Our results may also have relevance to dilute two dimensional Josephson junction arrays.

\section{Acknowledgments}

We would like to thank Dr. S.A. Leonel and Dr. P.Z. Coura for helpful discussions. This work was supported by CNPq (Brazilian agency).
[1] S. Villain-Guillot, R. Dandoloff, A. Saxena, Phys. Lett. A 188 (1994) 343.

[2] R. Dandoloff, S. Villain-Guillot, A. Saxena, A. R. Bishop, Phys. Rev. Lett. 74 (1995) 813.

[3] R. Dandoloff, A. Saxena, Eur. Phys. J. B 29 (2002) 265.

[4] S. Villain-Guillot, R. Dandoloff, A. Saxena, A.R. Bishop, Phys. Rev.B 52 (1995) 6712.

[5] J.M. Caillol, J. Physique-Lettres 42,(1981) L245.

[6] P.J. Forester, B. Jancovici, J. Stat. Phys. 84, (1996) 337.

[7] A. Krishnan, E. Dujardin, M.M.J. Treacy, J. Hugdahl, S. Lynum, T.W. Ebbesen, Nature (London) 388 (1997) 451.

[8] P.E. Lammert, V.H. Crespi, Phys. Rev. Lett. 85 (2000).

[9] J.D. Brown, Lower Dimensional Gravity (World Scientific, New Jersey, 1988), and references therein.

[10] A.R. Pereira, A.S.T. Pires, J. Magn. Magn. Mater. 257 (2003) 290.
[11] L.A.S. Mól, A.R. Pereira, W.A. Moura-Melo, Phys. Rev. B 67 (2003) 132403.

[12] A.R. Pereira, Phys. Lett. A 314 (2003) 102.

[13] A.R. Pereira, L.A.S. Mól, S.A. Leonel, P.Z. Coura, B.V. Costa, Phys. Rev. B 68 (2003) 132409.

[14] J.M. Kosterlitz, D.J. Thouless, J. Phys. C 6 (1973) 1181.

[15] F.G. Mertens, A.R. Bishop, G.M. Wysin, C. Kawabata, Phys. Rev. B 39 (1989) 591.

[16] A.R. Pereira, A.S.T. Pires, M.E. Gouvea, B.V. Costa, Z. Phys. B 39 (1992) 109.

[17] M.E. Gouvea, G.M. Wysin, A.R. Bishop, F.G. Mertens, Phys. Rev. B 39 (1989) 11840.

[18] G.M. Wysin, Phys. Rev. B 49 (1994) 8780.

[19] G.M. Wysin, Phys. Rev. B 54 (1996) 15156.

[20] H. Kawamura, M. Kikuchi, Phys. Rev. B 47 (1993) 1134.

[21] H.G. Evertz, D.P. Landau, Phys. Rev. B 54 (1996) 12302. 